\title{
Suramin Inhibits the Development and Progression of Peritoneal Fibrosis $\$$
}

\author{
Chongxiang Xiong, Na Liu, Lu Fang, Shougang Zhuang, and Haidong Yan \\ Department of Nephrology, Shanghai East Hospital, Tongji University School of Medicine, Shanghai, China (C.X., N.L., L.F., S.Z., \\ H.Y.); and Department of Medicine, Rhode Island Hospital and Brown University School of Medicine, Providence, Rhode Island \\ (S.Z.)
}

Received April 19, 2014; accepted August 27, 2014

\begin{abstract}
Peritoneal fibrosis is one of the most serious complications in patients with peritoneal dialysis (PD) and is associated with the loss of peritoneal membrane ultrafiltration function. In this study, we investigated whether suramin, an inhibitor that blocks multiple growth factors by binding to their receptors, would prevent development of peritoneal fibrosis in a rat model. Rats were given a daily intraperitoneal injection of chlorhexidine gluconate (CG) for 3 weeks to induce peritoneal fibrosis. Administration of suramin at 5,10 , and $20 \mathrm{mg} / \mathrm{kg}$ dose-dependently attenuated peritoneal membrane thickening and expression of collagen I, fibronectin, and $\alpha$-smooth muscle actin. Increased expression of transforming growth factor- $\beta 1$ (TGF- $\beta 1$ ) and phosphorylation of Smad3 was
\end{abstract}

detected in fibrotic peritoneum and inhibited by suramin treatment. Suramin was also effective in blocking CG-induced phosphorylation of inhibitor of $\kappa \mathrm{B}\left(\mathrm{I}_{\kappa} \mathrm{B}\right)$ and nuclear factor (NF)- $\kappa \mathrm{Bp} 65$, expression of several inflammatory cytokines, and infiltration of macrophages in the peritoneum. Moreover, suramin suppressed angiogenesis and expression of vascular endothelial growth factor, a molecule associated with angiogenesis in the injured peritoneum. Therefore, our results indicate that suramin treatment can effectively alleviate the development of peritoneal fibrosis by suppression of TGF- $\beta 1$ signaling, inflammation, and angiogenesis, and suggest that suramin may have therapeutic potential for prevention of peritoneal fibrosis in PD patients.

\section{Introduction}

Peritoneal dialysis (PD) is one of the renal replacement therapies used in patients with end-stage renal disease (Chaudhary et al., 2011). During the process of PD, the peritoneal membrane is continuously exposed to a variety of insults, including bioincompatible solutions, peritonitis, uremia, and chronic inflammation (Blake et al., 2013). Long-term exposure to these insults often causes peritoneal injury and subsequently leads to peritoneal fibrosis (Kaneko et al., 2007). As peritoneal fibrosis is closely associated with the decline of peritoneal function and ultimately leads to ultrafiltration and solute transport failure (Nakamura and Niwa, 2004), it is important to develop interventions for prevention and treatment of peritoneal fibrosis.

Peritoneal fibrosis is characterized by reduction or loss of mesothelial cells, changes in the structure and number of blood vessels, and enlargement of the submesothelial compact zone because of interstitial fibrosis (Tomino, 2012; de Lima et al.,

This study was supported by The National Nature Science Foundation of China [Grants 81170638 (to H.Y.), 81270778 and 81470920 (to S.Z.), 81200492 and 81470991 (to N.L.)]; the Shanghai Scientific Committee of China [Grant 13PJ1406900 (to N.L.)] and by Key Discipline Construction Project of Pudong Health Bureau of Shanghai [Grant PWZx2014-06 (to S.Z.)].

C.X. and N.L. are co-first authors.

dx.doi.org/10.1124/jpet.114.215228.

S This article has supplemental material available at jpet.aspetjournals.org.
2013). Activation of fibroblasts with expression of $\alpha$-smooth muscle actin ( $\alpha$-SMA) is the cellular basis of interstitial fibrosis, which is accompanied by excessive deposition of extracellular matrix components, such as collagen I and fibronectin. Although the pathophysiologic role of fibroblasts in the peritoneum remains poorly understood, multiple factors and mechanisms, such as inflammation (Tomino, 2012; de Lima et al., 2013), neoangiogenesis (Sekiguchi et al., 2012), and activation of transforming growth factor- $\beta 1$ (TGF- $\beta 1$ ) signaling (Chegini, 2008; Liu et al., 2012), have been shown to contribute to this process. TGF- $\beta 1$ exerts its biologic actions through activation of two downstream signaling molecules, Smad2/3. Previous studies have indicated an increase in the expression of TGF- $\beta 1$ and phosphorylation of Smad2/3 in the peritoneum in a rat model of chlorhexidine digluconate-induced peritoneal fibrosis (Lee et al., 2014). It is also evident that TGF- $\beta 1$ causes peritoneal injury through a Smad-dependent signaling pathway (Patel et al., 2010). These data suggest that TGF- $\beta$ signaling plays an important role in the development of PD.

Inflammation is also critically connected with the progression of peritoneal fibrosis (Lai and Leung, 2010). After peritoneal injury, a variety of cytokines/chemokines, such as interleukin-1 (IL-1), IL-6, tumor necrosis factor- $\alpha$ (TNF- $\alpha$ ), and monocyte chemotactic protein-1 (MCP-1), are produced, and multiple leukocytes, in particular macrophages, are recruited to the injured

ABBREVIATIONS: CG, chlorhexidine gluconate; DAB, 3,3'-diaminobenzidine (3,3',4,4'-tetraamino-diphenyl); ECM, extracellular matrix; ELISA, enzyme-linked immunosorbent assay; GAPDH, glyceraldehyde 3-phosphate dehydrogenase; I $\mathrm{B}$, inhibitor of $\kappa \mathrm{B}$; IL, interleukin; MCP-1, monocyte chemotactic protein-1; NF- $\kappa \mathrm{B}$, nuclear factor- $\kappa \mathrm{B}$; PD, peritoneal dialysis; $\alpha$-SMA, $\alpha$-smooth muscle actin; TGF- $\beta 1$, transforming growth factor- $\beta 1$; TNF- $\alpha$, tumor necrotic factor- $\alpha$; VEGF, vascular endothelial growth factor. 
area. In addition, nuclear factor $-\kappa \mathrm{B}(\mathrm{NF}-\kappa \mathrm{B})$ has been considered an important proinflammatory transcriptional factor (Muriel, 2009). Under normal circumstances, I $\kappa \mathrm{Bs}$, inhibitory proteins, constrain movement of NF- $\kappa \mathrm{B}$ dimers into the nucleus. During the inflammatory process, different stimuli induce ubiquitination of phosphorylated $I_{\kappa} B s$ and their subsequent degradation by the proteasome (Herrmann et al., 2004). I $\kappa$ B degradation allows $\mathrm{NF}-\kappa \mathrm{B}$ to translocate into the nucleus (Karin, 2009), where it leads to the transcription of many genes involved in inflammatory process (Hoesel and Schmid, 2013). Inflammatory targets regulated by $\mathrm{NF}-\kappa \mathrm{B}$ include chemokines, cytokines, and adhesion molecules (Sun et al., 2004; Huang et al., 2013).

Long-term PD often causes progressive angiogenesis, an event associated with peritoneal fibrosis and ultrafiltration failure of the peritoneal membrane (Stavenuiter et al., 2011). As vascular endothelial growth factor (VEGF) is a potent proangiogenic cytokine (Fusshoeller, 2008; Saxena, 2008), several studies have examined the possible involvement of VEGF in peritoneal fibrosis. For example, expression of VEGF in mesothelial cells that have undergone epithelial-to-mesenchymal transition is increased in PD patients (Aroeira et al., 2005); a large number of VEGFpositive cells were observed in the thickening submesothelial area (Yoshio et al., 2004); and treatment with a neutralizing antiVEGF monoclonal antibody was able to prevent structural and functional microvascular alterations induced by hyperglycemia (De Vriese et al., 2001). As such, inhibition of VEGF expression may be an interesting therapeutic approach for prevention of peritoneal fibrosis.

Suramin is a polysulfonated naphthylurea that was originally used in the prevention and treatment of early stages of human trypanosomiasis; it also has antitumor activity (Liu et al., 2011).
Suramin exerts its pharmacologic effect through the interaction of several cytokines and growth factors with their receptors. Mechanistic studies demonstrate that suramin inhibits apoptosis, suppresses expression of proinflammatory cytokines, inactivates myofibroblasts, and blocks multiple cell signaling pathways (Liu and Zhuang, 2011). Recent animal studies from our laboratory and by other investigators have shown that administration of suramin can attenuate muscle, liver, and renal fibrosis (Chan et al., 2003; Li et al., 2009; Liu et al., 2011). We have shown that suramin treatment can suppress the expression of fibronectin and $\alpha$-SMA and type I collagen and reduce the deposition of extracellular matrix (ECM).

Currently, it is unknown whether suramin has an antifibrotic effect in the peritoneum. In this study, we investigated the effect of suramin on the development of peritoneal fibrosis induced by chlorhexidine gluconate (CG) and the mechanisms involved in rats.

\section{Materials and Methods}

Antibodies and Chemicals. Antibodies to p-I $\kappa \mathrm{B}, \mathrm{I} \kappa \mathrm{B}, \mathrm{p}-\mathrm{NF}-\kappa \mathrm{B}$, $\mathrm{NF}-\kappa \mathrm{B}, \mathrm{p}-\mathrm{Smad} 3$, and Smad3 were purchased from Cell Signaling Technologies (Danvers, MA). Antibodies to fibronection, collagen I (A2), and Smad7 were purchased from Santa Cruz Biotechnology, Inc. (Dallas, TX). Antibodies to GAPDH (glyceraldehyde 3-phosphate dehydrogenase), VEGF, and CD68 were purchased from BD Pharmingen (San Diego, CA). TNF- $\alpha$, IL-1 $\beta$, MCP-1, IL-6, TGF- $\beta 1$ enzyme-linked immunosorbent assay (ELISA) kits were from R\&D Systems (Minneapolis, MN). Suramin, $\alpha$-SMA, CG, and all other chemicals were from Sigma-Aldrich (St. Louis, MO).

Creation of the Rat Model with Peritoneal Fibrosis and Suramin Treatment. Thirty-six 6- to 8-week-old male Sprague-Dawley
A

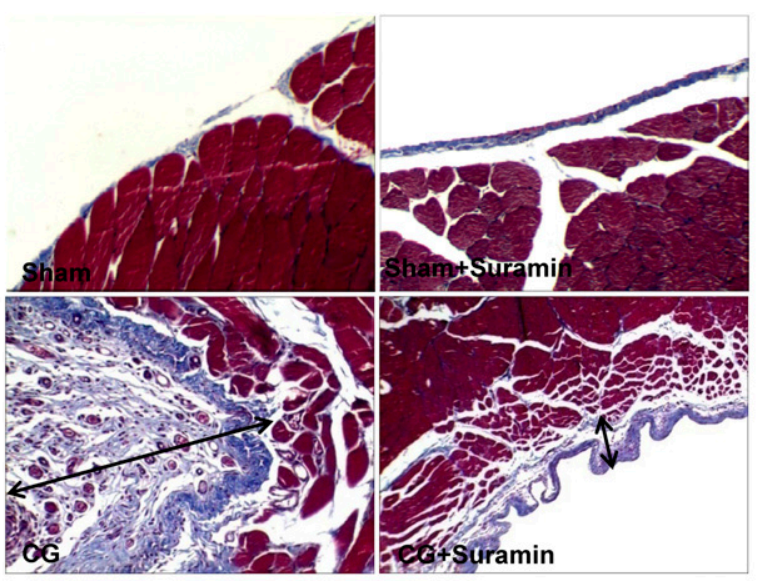

B

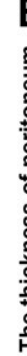

Trichrome Masson Staining

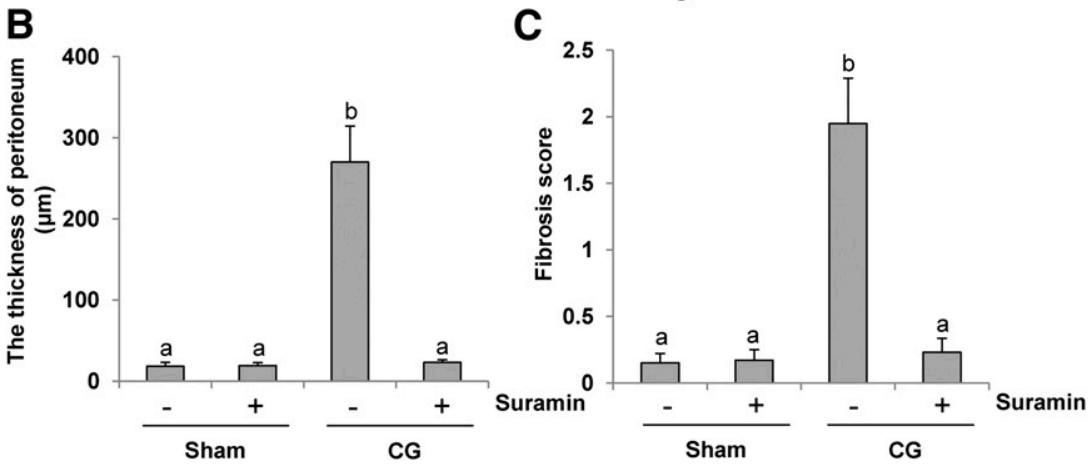

Fig. 1. Suramin attenuates CG-induced peritoneal fibrosis in rats. Photomicrographs illustrating Masson trichrome staining of peritoneum in the rat model of GC-induced peritoneal fibrosis administered with/without $20 \mathrm{mg} / \mathrm{kg}$ suramin (A); thickening of the compact zone (B); and the score of fibrosis (C) of peritoneal tissue. Data are represented as the mean \pm S.E.M. $(n=6)$. Means with different lowercase letters are significantly different from one another $(P<0.05)$. 
rats $(170 \pm 10 \mathrm{~g})$ were purchased from Shanghai Super-B\&K Laboratory Animal Corp. Ltd. (Shanghai, China). Animals were housed in stainless steel cages in a ventilated animal room at the Experimental Animal Center of Tongji University. Room temperature was maintained at $20 \pm 2^{\circ} \mathrm{C}$, relative humidity at $60 \pm 10 \%$, and a 12 -hour light/dark cycle. Distilled water and sterilized food for rats were available ad libitum. Animals were acclimated to this environment for 7 days prior to experiments. Rats were randomly assigned to six groups with six rats per group: the sham group, sham treated with suramin $(20 \mathrm{mg} / \mathrm{kg})$ group, the peritoneal fibrosis group, and rats with peritoneal fibrosis treated with different doses of suramin $(5,10,20 \mathrm{mg} / \mathrm{kg}$, respectively). The peritoneal fibrosis in rats was generated by daily intraperitoneal injection of $0.1 \%$ gluconate chlorhexidine $(1 \mathrm{ml} / 100 \mathrm{~g})$ as described previously with a slight modification (Bozkurt et al., 2008). Normal saline $(0.9 \%)$ was administered as a control. In the treatment groups, suramin was intraperitoneally injected when the first dose of gluconate chlorhexidine was administered and then given weekly at $5,10,20 \mathrm{mg} / \mathrm{kg}$. At the end of 3 weeks, all rats were sacrificed and the parietal peritoneum away from the injection points was harvested for further analysis. All experiments were conducted in accordance with the animal experimentation guideline of Tongji University School of Medicine.

Morphologic Studies of Peritoneum. Tissue sections were prepared at $4-\mu \mathrm{m}$ thickness by a routine procedure followed by fixation in $4 \%$ paraformaldehyde, dehydration and clearance in conventional-series ethanols and xylene, and embedding in paraffin. Sections were stained with Masson's trichrome or H\&E for general histology, especially changes in the thickness of peritoneum. The thickness of the submesothelial tissue was evaluated (in micrometers), and the average of ten independent measurements was calculated for each section ( $400 \times$ magnification).

Immunoblot Analysis. Immunoblot analysis of peritoneum tissue samples were conducted as described previously (Pang et al.,
2009). The densitometry analysis of immunoblot results was conducted by using NIH ImageJ software (NIH, Bethesda, MD).

Immunohistochemical Staining. The tissue sections were treated for 30 minutes in methanol containing $30 \%$ hydrogen peroxide $\left(\mathrm{H}_{2} \mathrm{O}_{2}\right)$ after being routinely deparaffinized and hydrated. After washing with phosphate-buffered saline three times, microwave antigen retrieval was conducted in citric acid buffer ( $\mathrm{pH}$ 6.0) microwave, blocked with 5\% bovine serum albumin blocking buffer at $37^{\circ} \mathrm{C}$ for 20 minutes, and washed with phosphate-buffered saline three times. Sections were then incubated with primary antibodies at $4^{\circ} \mathrm{C}$ overnight and homologous secondary antibodies at room temperature.

Following DAB [3,3'-diaminobenzidine (3,3',4,4'-tetraamino-diphenyl)] coloration at room temperature, counterstaining in $\mathrm{H} \& \mathrm{E}$, and mounting with Neutral balsam, morphologic analyses were performed by using light microscopy. The antibody dilutions are: collagen-I antibody (1:250), anti- $\alpha$-SMA (1:200), fibronectin antibody (1:200), CD68 antibody (1:200), CD31 (1:200), and VEGF antibody (1:200). For quantitative assessment, 10 areas were selected and the positive staining area of collagen I and fibronectin was determined by Image Pro-Plus Software (NIH) and the average ratio to each microscopic field $(400 \times)$ was calculated and graphed. The numbers of $\alpha$-SMAexpressing cells, CD68-positive macrophages, CD31-positive vessels, and VEGF-positive cells and were calculated in 10 fields at $400 \times$ magnification.

ELISA Detection. ELISA detection of TGF- $\beta 1$, MCP-1, IL- $1 \beta$, TNF- $\alpha$, IL- 6 protein was performed in accordance with the manufacturer's instructions.

Statistical Analysis. All the experiments were conducted at least three times. Data depicted in graphs represent the means \pm S.E.M. for each group. Intergroup comparisons were made using one-way analysis of variance. Multiple means were compared using
A

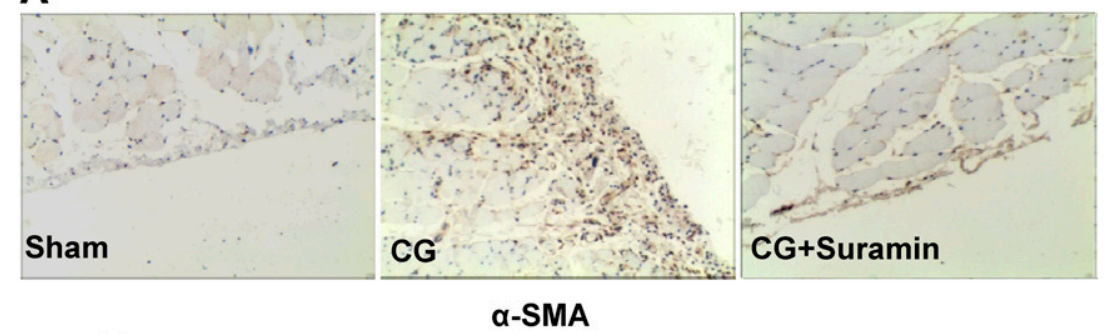

B
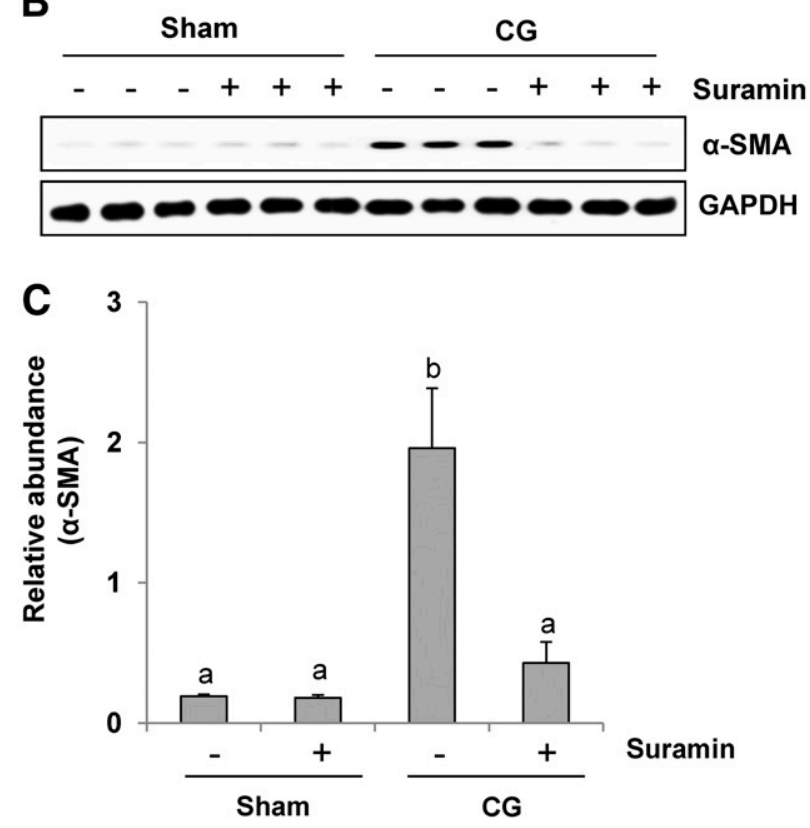

Fig. 2. Suramin inhibits $\alpha$-SMA expression in the rat model of CG-induced peritoneal fibrosis. Immunostaining of $\alpha$-SMA in the submesothelial compact zone (A). Peritoneal lysates were prepared and subject to immunoblot analysis with antibodies to $\alpha$-SMA or GAPDH (B). Representative immunoblots from three or more experiments are shown. (C) Expression levels of the proteins were quantified by densitometry and normalized with GAPDH. Data are represented as the mean \pm S.E.M. Means with different lowercase letters are significantly different from one another $(P<0.05)$. 
Tukey's test. The differences between two groups were determined by Student's $t$ test. Statistical significant difference between mean values was marked in each graph. $P<0.05$ is considered significant.

\section{Results}

Suramin Prevents Chlorhexidine Gluconate-Induced Peritoneal Fibrosis in Rats. We recently showed that administration of suramin alleviates progression of renal fibrosis in a mouse model of unilateral ureteral obstruction (Liu et al., 2011). However, the pharmacological effect of suramin in peritoneal fibrosis remains unclear. The pathogenesis of peritoneal fibrosis is characterized by overproduction and deposition of extracellular matrix, which leads to fibrotic lesions and peritoneal scarring. Thus, we first examined the effect of suramin on the expression of peritoneal collagen fibrils by Masson's trichrome staining. Rat peritoneal fibrosis was established by daily injection of CG for 3 weeks. Suramin treatment started immediately after injection of CG at concentrations of $5,10,20 \mathrm{mg} / \mathrm{kg}$, which was then administered weekly until animals were sacrificed. Peritoneum administered with CG displayed severe morphologic lesions characterized by the thickening of submesothelial compact zone, peritoneal mesothelial cell loss, and peritoneal interstitial expansion with collagen accumulation and deposition. Treatment with suramin reduced the thickness of peritoneum in a dose-dependent manner with maximum effect at $20 \mathrm{mg} / \mathrm{kg}$
(Fig. 1, A and B; Supplemental Figs. 1 and 2). The quantitative analysis of thickness of peritoneum indicated an 8-fold increase in chlorhexidine gluconate-injured rats compared with sham-operated animals. Suramin treatment dose dependently attenuated this fibrotic response: at $20 \mathrm{mg} / \mathrm{kg}$, suramin was sufficient to prevent it (Fig. 1C). These data suggest that suramin is a potent agent in preventing the development of peritoneal fibrosis.

Suramin Inhibits Expression of $\alpha$-SMA in the Rat Model of Chlorhexidine Gluconate-Induced Peritoneal Fibrosis. To investigate the ability of suramin in suppressing peritoneal myofibroblast activation, we examined the effect of suramin on the expression of $\alpha$-SMA, a hallmark of myofibroblasts in the peritoneum. Western blot and immunohistochemistry analysis of peritoneum showed the marked upregulation of $\alpha$-SMA expression in the rat model of peritoneal fibrosis, and administration of suramin significantly reduced this response (Fig. 2). Inhibition of $\alpha$-SMA expression by suramin also occurred in a dose-dependent manner, with the observed effect at $5 \mathrm{mg} / \mathrm{kg}$, a greater inhibition at $10 \mathrm{mg} / \mathrm{kg}$, and the maximum effect at $20 \mathrm{mg} / \mathrm{kg}$ (Fig. 2, A and B; Supplemental Fig. 1). Suramin administration suppressed peritoneal expression of $\alpha$-SMA by approximately $75 \%$ in chlorhexidine gluconate-treated animals compared with those treated with chlorhexidine gluconate alone (Fig. $2 \mathrm{C})$. This suggests that suramin has a potent capability to inhibit activation of peritoneal fibroblasts.
A

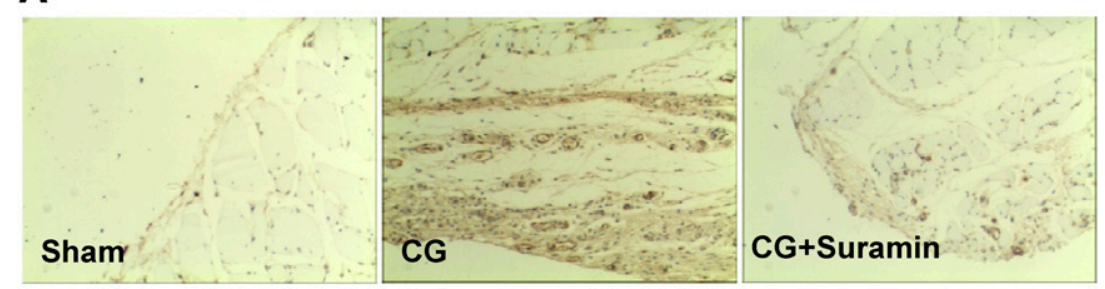

Fibronectin
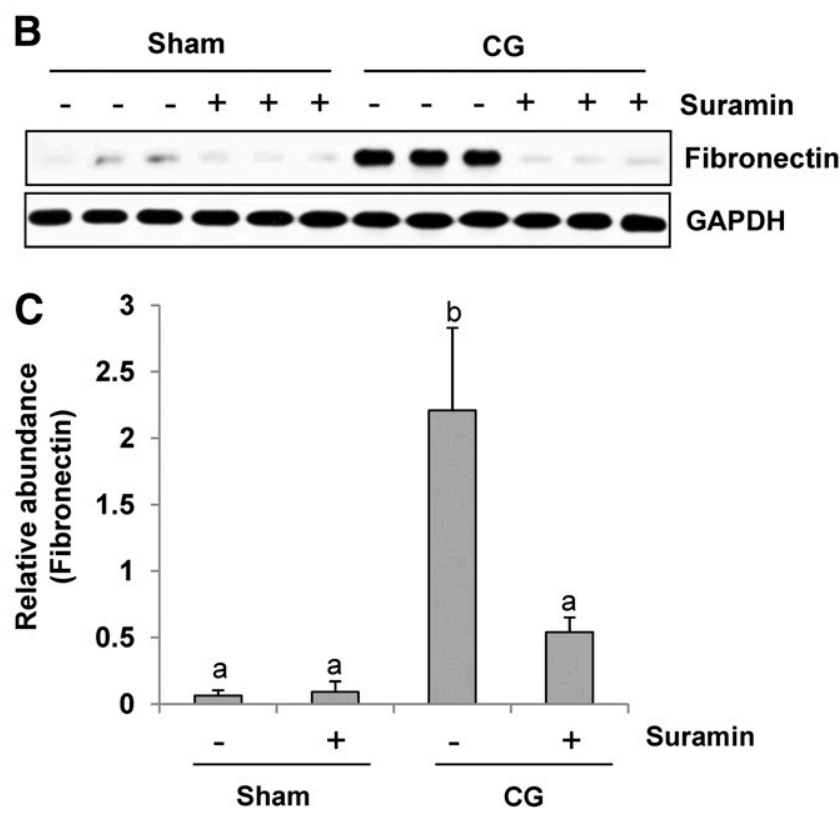

Fig. 3. Suramin blocks fibronection expression in the rat model of CG-induced peritoneal fibrosis. Immunostaining of fibronectin in the submesothelial compact zone (A). Peritoneal tissue lysates were subjected to immunoblot analysis with specific antibodies against fibronectin (B). Expression levels of fibronectin were quantified by densitometry and normalized with GAPDH (C). Data are represented as the mean \pm S.E.M. $(n=6)$. Means with different lowercase letters are significantly different from one another $(P<0.05)$. 
Suramin Blocks Expression of Fibronectin and Collagen I in the Rat Model of Chlorhexidine GluconateInduced Peritoneal Fibrosis. Tissue fibrosis is characterized by accumulation of ECM components, such as fibronectin and collagen I, two major ECM proteins (Liu et al., 2011; Subeq et al., 2011). As such, we examined the effect of suramin on their expression in rats with peritoneal fibrosis. Immunohistochemistry staining and Western blot analysis of peritoneum indicated increased expression of fibronectin (Fig. 3) and collagen I (Fig. 4) in this model. Suramin treatment also dose dependently reduced their expression with the significant inhibition at $5 \mathrm{mg} / \mathrm{kg}$, and to the basal level when $20 \mathrm{mg} / \mathrm{kg}$ was used (Supplemental Fig. 1). Quantitative analysis of Western blotting results indicates that treatment with $20 \mathrm{mg} / \mathrm{kg}$ suramin reduced the expression of fibronectin and collagen I by approximately 80 and $70 \%$, respectively, in chlorhexidine gluconate-treated rats (Figs. 3C and 4C). Collectively, these results indicate that suramin treatment suppresses expression of ECM proteins.

Suramin Inhibits TGF- $\beta 1$ Expression and Smad3 Phosphorylation in the Rat Model of Chlorhexidine Gluconate-Induced Peritoneal Fibrosis. TGF- $\beta$ signaling pathway is associated with most fibrotic diseases, and an increase in its markers has been considered as a major mechanism of peritoneal fibrosis (Liu, 2006; Wynn, 2008). To investigate the effect of suramin on the TGF- $\beta$ signaling in the rat model of peritoneal fibrosis, we first examined the expression level of TGF- $\beta 1$ in the peritoneum by ELISA. Figure 5A shows that chlorhexidine gluconate injury induced a marked increase in the expression of TGF- $\beta 1$, and administration of suramin reduced its expression by $50 \%$. We next examined the effect of suramin on the phosphorylation of Smad3 and expression of Smad7, two key mediators in TGF- $\beta$ signaling (Bhogal et al., 2005; Kaminska et al., 2005). Chlorhexidine gluconate injury resulted in increased phosphorylation of Smad3. Treatment with suramin reduced Smad3 phosphorylation (Fig. 5, B and D), and increased expression of Smad7 (Fig. 5, C and D). Expression of total Smad3 was not affected by chlorhexidine gluconate and suramin (Fig. 5B). As decreased Smad3 phosphorylation and increased expression of Smad7 have been shown to be associated with inhibition of peritoneal fibrosis progression in an experimental rat model, we suggest that inhibition of TGF- $\beta$ signaling may be largely associated with the antifibrotic effect of suramin.

Suramin Inhibits NF-кB Signaling in the Rat Model of Chlorhexidine Gluconate-Induced Peritoneal Fibrosis. Activation of NF- $\kappa \mathrm{B}$ signaling pathway is a key regulator of angiogenesis and inflammation in peritoneal fibrosis (Kitamura et al., 2012). $\mathrm{I} \kappa \mathrm{B}$ is the inhibitor of NF- $\kappa \mathrm{B}$ (Man and Zhang, 2011). To examine the effect of suramin on the $\mathrm{NF}-\kappa \mathrm{B}$ pathway in peritoneal fibrosis, experiments were conducted to analyze total $I_{\kappa} B$ and phosphorylated $I_{\kappa} B$ levels by immunoblot analysis. Chlorhexidine gluconate injury induced $\mathrm{I}_{\kappa} \mathrm{B}$ phosphorylation in the peritoneum, which was
A

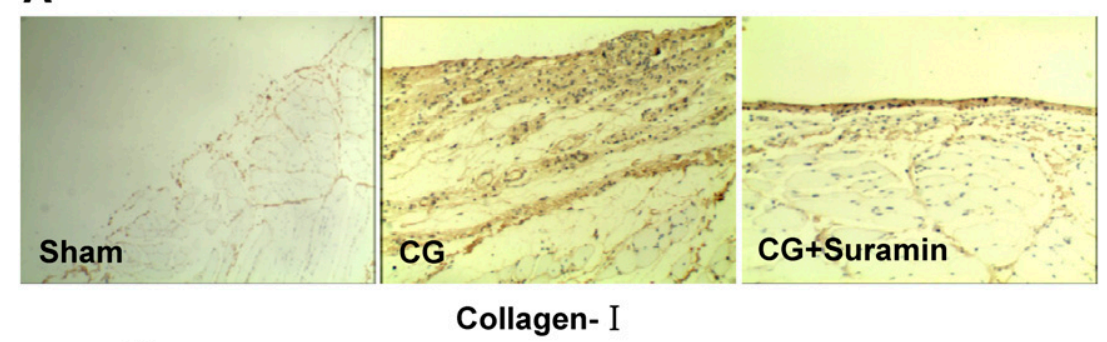

B
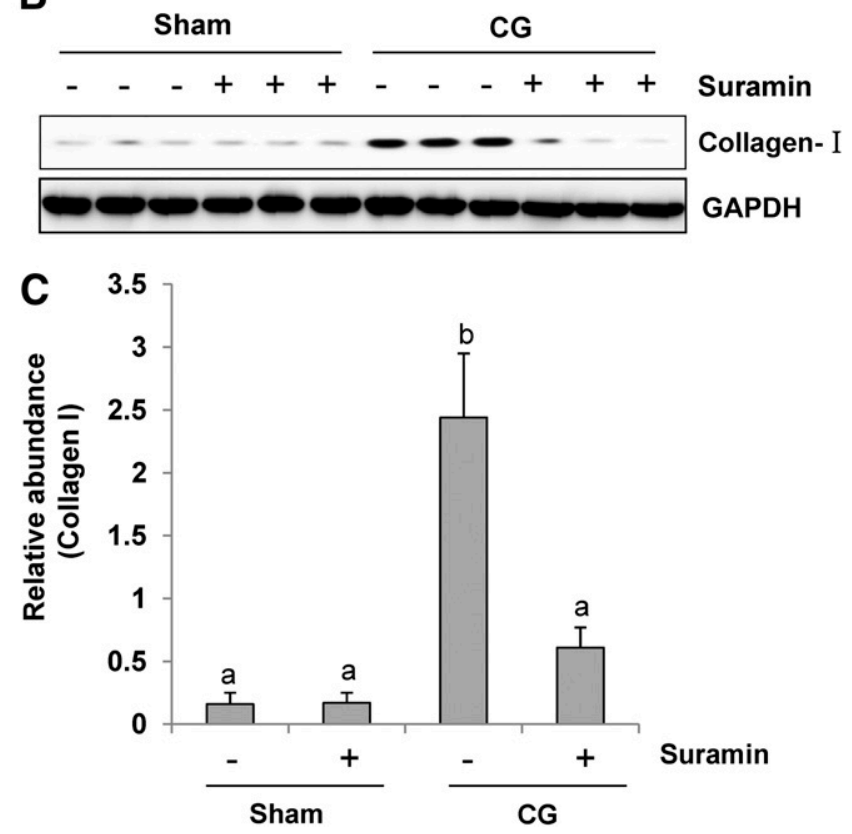

Fig. 4. Suramin blocks collagen I expression in the rat model of CG-induced peritoneal fibrosis. Immunostaining of collagen I in the submesothelial compact zone (A). Peritoneal tissue lysates were subjected to immunoblot analysis with specific antibodies against collagen I (B). Expression levels of collagen I were quantified by densitometry and normalized with GAPDH (C). Data are represented as the mean \pm S.E.M. $(n=6)$. Means with different lowercase letters are significantly different from one another $(P<0.05)$. 


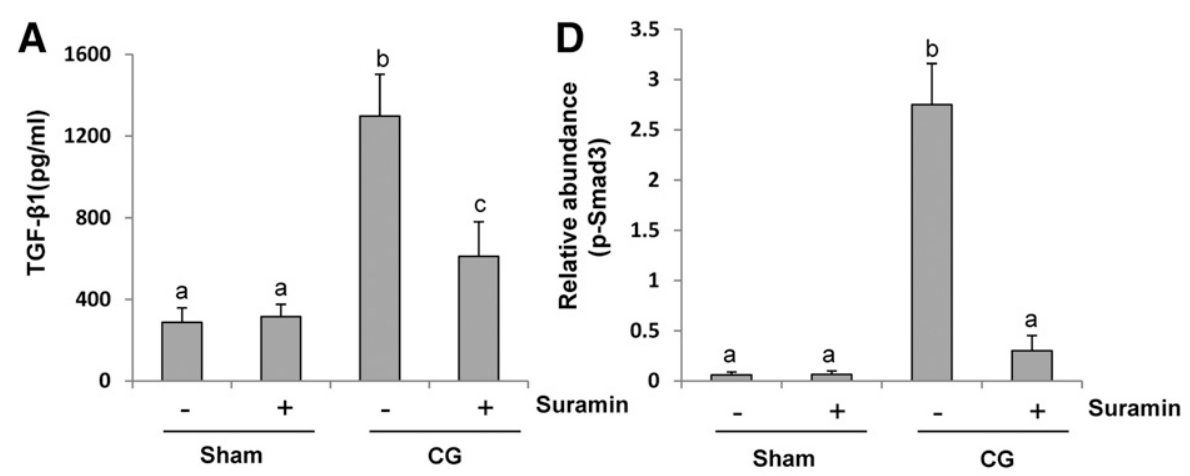

B

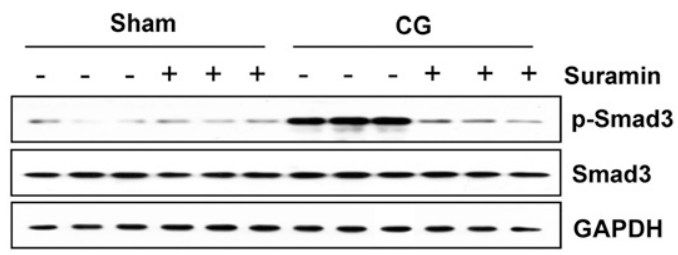

C

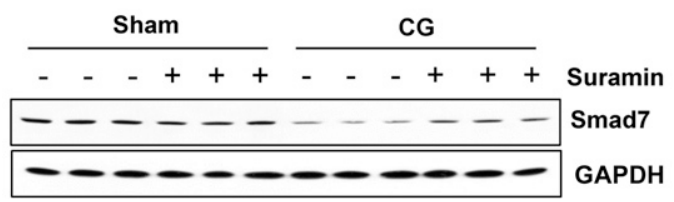

inhibited in the presence of suramin (Fig. 6). The phosphorylated $\mathrm{I} \kappa \mathrm{B}$ was not detected in the peritoneum of sham and sham-treated animals with suramin.

Next, we examined the effect of suramin on the expression of phosphorylated NF- $\kappa$ Bp 65 . The phosphorylated NF- $\kappa$ Bp 65 was barely detected in the peritoneum of sham and sham-treated suramin rats but its expression level was significantly upregulated in the rats with peritoneal fibrosis. Administration of suramin significantly inhibited NF- $\kappa \mathrm{Bp} 65$ phosphorylation without affecting expression of its total levels (Fig. 7, A and B). These data suggest that suramin is effective in inhibiting activation of $\mathrm{NF}-\kappa \mathrm{B}$ signaling pathway in the peritoneum.

Suramin Suppresses Expression of Multiple Inflammatory Cytokines in Chlorhexidine Gluconate-Induced Peritoneal Fibrosis in Rats. Proinflammatory cytokines and adhesion molecules play an important role in the development and progression of peritoneal fibrosis. To determine the effect of suramin on inflammatory cytokines, we measured their expression levels in rats with peritoneal fibrosis, treated or untreated with suramin, by ELISA (Fig. 8). The expression levels of proinflammatory cytokines and chemokines, including IL- $6,-\alpha$, MCP-1, and IL- $1 \beta$, were significantly increased in rats with peritoneal fibrosis. Suramin treatment decreased their expression levels (Fig. 8, A-D).

Suramin Inhibits Infiltration of Macrophages in Chlorhexidine Gluconate-Induced Peritoneal Fibrosis in Rats. One of the characteristic pathologic changes in peritoneal fibrosis is the infiltration of macrophages into the thickened submesothelial compact zone (Nishino et al., 2012). Reduced macrophage infiltration would have a potential for preventing peritoneal fibrosis. To elucidate suramin on this process, we conducted immunohistochemistry staining using an antibody against CD68, a marker of active macrophages.
Fig. 5. Suramin inhibits expression of TGF- $\beta 1$ and Smad3 phosphorylation in the rat model of CG-induced peritoneal fibrosis. Protein from peritoneal tissues from sham-operated or CG-treated rats with/without suramin administration was subjected to the determination of TGF- $\beta 1$ levels by the ELISA (A). Peritoneal lysates were subject to immunoblot analysis with antibodies to phosphorylated Smad3, Smad3, or GAPDH (B) as well as Smad7 or GAPDH (C). Expression levels of p-Smad3 were quantified by densitometry and normalized with total Smad3 (D). Data are represented as the mean \pm S.E.M. $(n=6)$. Means with different lowercase letters are significantly different from one another $(P<0.05)$.

Figure 9A demonstrated that macrophage infiltration was increased in the peritoneum of rats after chlorhexidine gluconate injection. Compared with the sham animals, suramin treatment
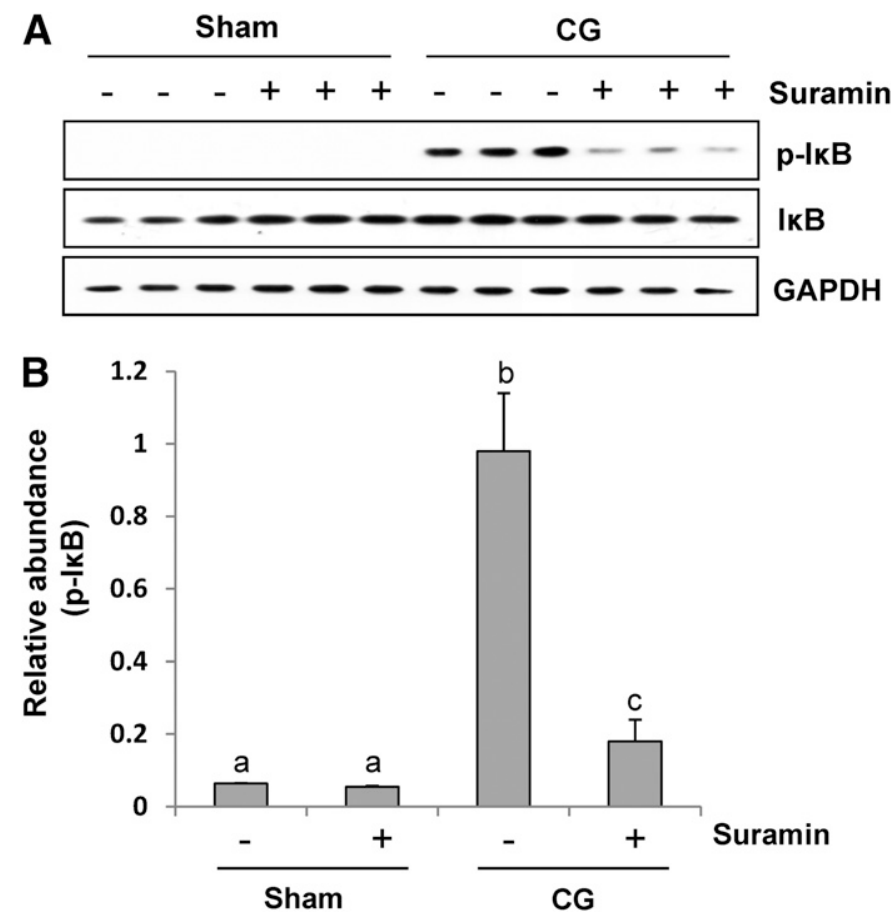

Fig. 6. Suramin inhibits $I-\kappa B$ phosphorylation in the rat model of CG-induced peritoneal fibrosis. Peritoneal tissue lysates were subjected to immunoblot analysis with specific antibodies against phospho-I $\kappa \mathrm{B}$ $(\mathrm{p}-\mathrm{I} \kappa \mathrm{B}), \mathrm{I} \kappa \mathrm{B}$, or GAPDH (A). Expression levels of $\mathrm{p}-\mathrm{I} \kappa \mathrm{B}$ were quantified by densitometry and normalized with $\mathrm{I} \kappa \mathrm{B}(\mathrm{B})$. Data are represented as the mean \pm S.E.M. $(n=6)$. Means with different lowercase letters are significantly different from one another $(P<0.05)$. 
A Sham CG

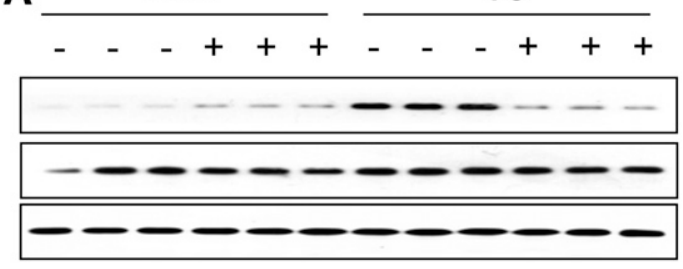

Suramin p-NFkB(p65) $\mathrm{NF} K \mathrm{~B}(\mathrm{p} 65)$ GAPDH

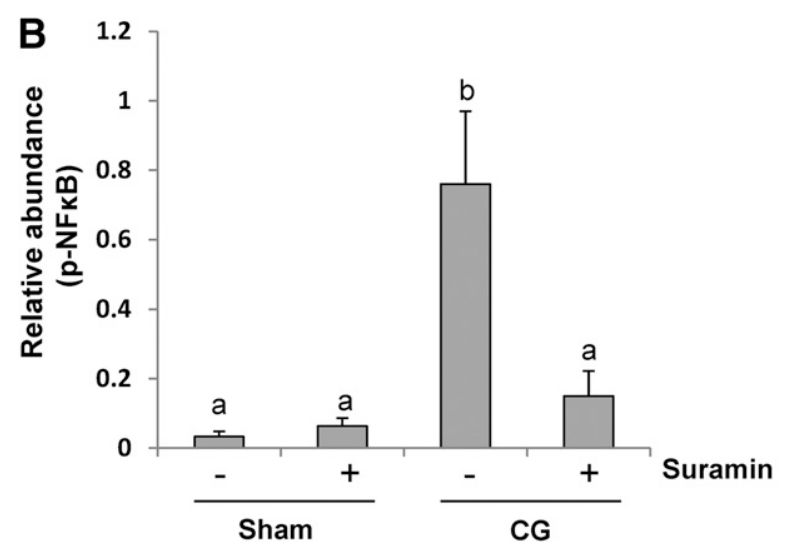

Fig. 7. Suramin inhibits NF- $\kappa$ B phosphorylation in the rat model of CG-induced peritoneal fibrosis. Peritoneal tissue lysates were subjected to immunoblot analysis with specific antibodies against phospho-NF- $\kappa \mathrm{B}$ p65, $\mathrm{NF}-\kappa \mathrm{B}$ p65 and GAPDH (A). Expression levels of phospho-NF- $\kappa$ Bp65 were quantified by densitometry and normalized with NF- $\kappa$ Bp65 (B). Data are represented as the mean \pm S.E.M. $(n=6)$. Means with different lowercase letters are significantly different from one another $(P<0.05)$.

significantly reduced the number of macrophages in the injured peritoneum (Fig. 9A). The quantitative analysis indicated that the number of CD68-postive macrophages was significantly increased in the rat with peritoneal fibrosis (Fig. 9B), and suramin treatment significantly reduced macrophage infiltration in the peritoneum. Therefore, suramin is also able to inhibit infiltration of macrophage cells into the submesothelial layer.

Suramin Decreases VEGF Expression in Chlorhexidine Gluconate-Induced Peritoneal Fibrosis in Rats. Long-term PD is frequently accompanied by neo-angiogenesis with vasculopathy (Ro et al., 2007). Previous studies have shown that the expression level of VEGF is elevated in the peritoneal membrane of $\mathrm{PD}$ patients, leading to angiogenesis of the peritoneum (Fusshoeller, 2008; Saxena, 2008). We thus also examined expression of VEGF and CD31 (a marker of vascular endothelial cells) by immunohistochemistry staining. Angiogenesis was evaluated by counting the number of CD31-positive blood vessels in the peritoneal tissue injured by chlorhexidine gluconate. A marked increase in VEGF-positive cells and CD31-positive blood vessels was observed, which was inhibited by suramin administration (Fig. 10, A and B). These data suggest that suramin may also prevent peritoneal fibrosis through suppression of neo-angiogenesis.

\section{Discussion}

In this study, we report that suramin can prevent the progression of peritoneal fibrosis induced by chlorhexidine gluconate. Suramin significantly inhibited thickening of the submesothelial compact zone, expression of $\alpha$-SMA, and deposition of ECM proteins (i.e., type I collagen and fibronectin) in the peritoneum of rats with peritoneal fibrosis. Suramin treatment also reduced macrophage infiltration and expression of VEGF. Furthermore, suramin administration inhibited expression of multiple cytokines and chemokines in the injured peritoneum. These data are consistent with the inhibitory effect of suramin on renal fibrosis and suggest that
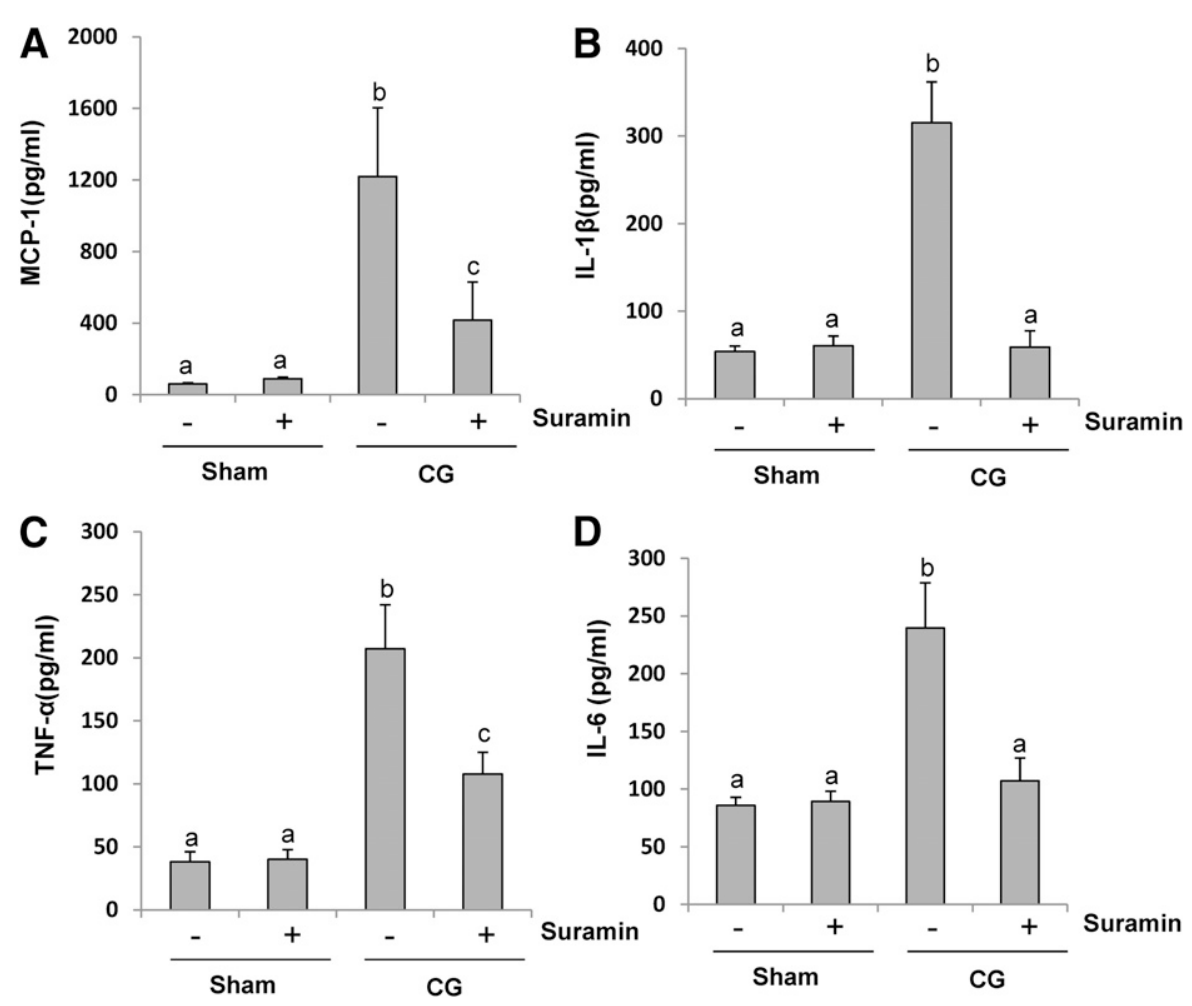

D

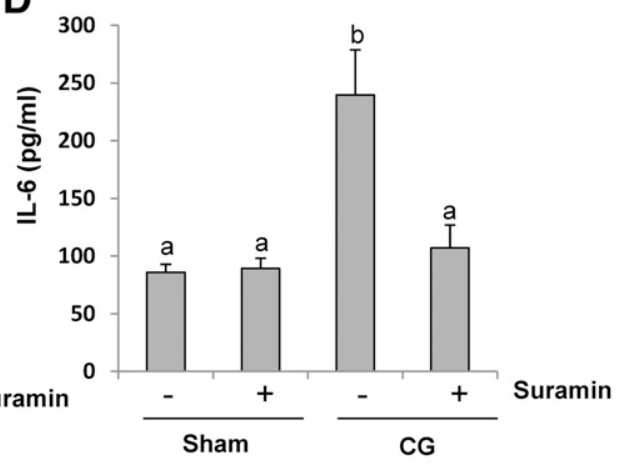

Fig. 8. Suramin suppresses the expression of MCP-1, TNF- $\alpha$, IL- $1 \beta$, and IL- 6 in the rat model of CG-induced peritoneal fibrosis. Peritoneal lysates were subjected to the ELISA as described under Materials and Methods. The expression levels of MCP-1 (A), IL-1 $\beta$ (B), TNF- $\alpha$ (C), and IL-6 (D) are indicated over control (sham with vehicle). Data are represented as the mean \pm S.E.M. $(n=6)$. Means with different lowercase letters are significantly different from one another $(P<0.05)$. 
A
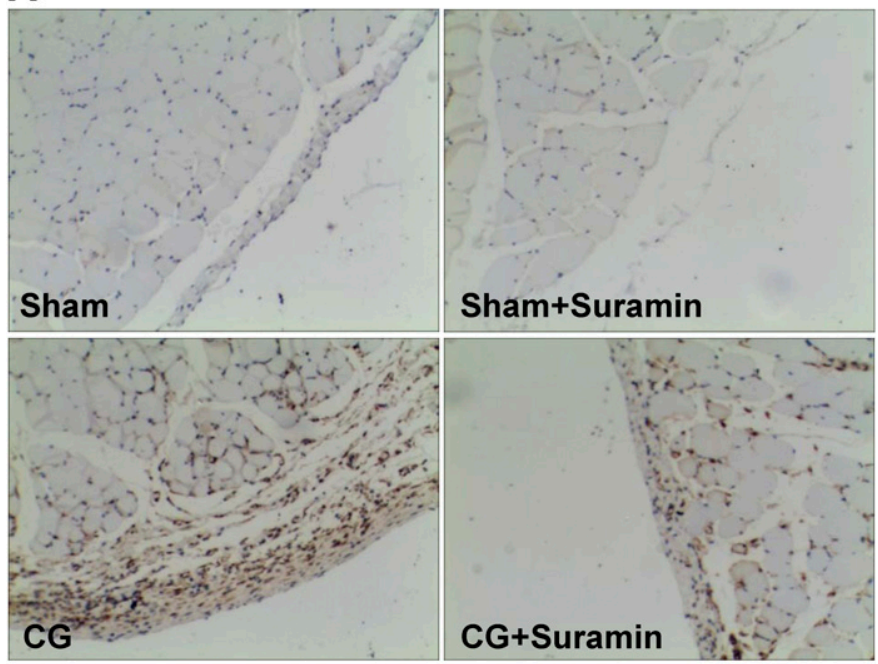

CD 68

B

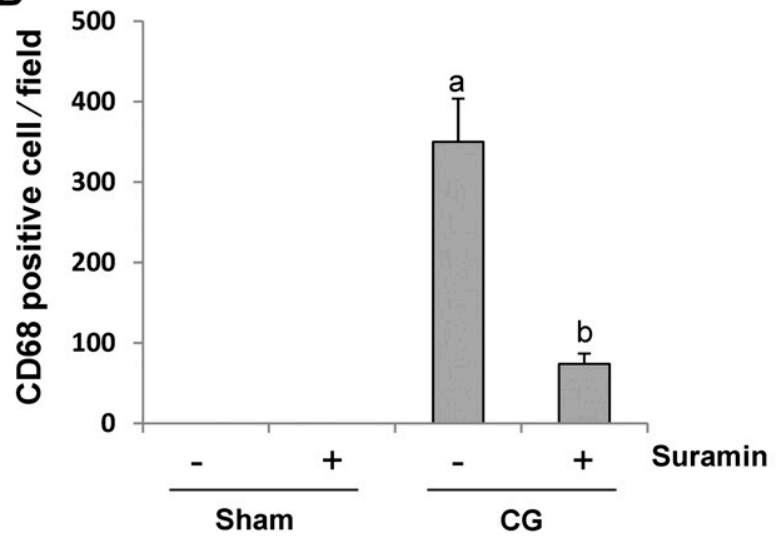

Fig. 9. Effect of suramin on the leukocyte infiltration in the peritoneum in the rat model of CG-induced peritoneal fibrosis. Immunostaining of leukocytes with CD68 in the submesothelial compact zone (A). Quantitative analysis was conducted in three random fields of each sample, and 10 fields $(1 \times 100)$ were analyzed for each condition. Data are represented as the mean \pm S.E.M. $(n=6)$. Means with different lowercase letters are significantly different from one another $(P<0.05)(\mathrm{B})$.

suramin is also a potent agent for attenuation of peritoneal fibrosis.

As in renal fibrogenesis, multiple signaling pathways are activated and involved in the development of peritoneal fibrosis. Of these pathways, TGF- $\beta$ signaling has been shown to play a predominant role in this process. To understand the mechanism by which suramin inhibits peritoneal fibrosis, we examined the effect of suramin on the expression of TGF- $\beta 1$ and activation of Smad3, a key mediator in TGF- $\beta$ signaling. Our results clearly showed that administration of suramin blocked TGF- $\beta 1$ expression as well as Smad3 phosphorylation. In addition, we also observed that suramin treatment increased expression of Smad7. These data, together with our previous observations that suramin is able to inhibit expression of ECM proteins and Smad3 phosphorylation in renal fibroblasts exposed to TGF- $\beta 1$ suggest that suramin may attenuate peritoneal fibrosis through suppression of the TGF- $\beta$ signaling pathway at different levels. However, this may not be the sole mechanism, as our previous studies also indicated that
A

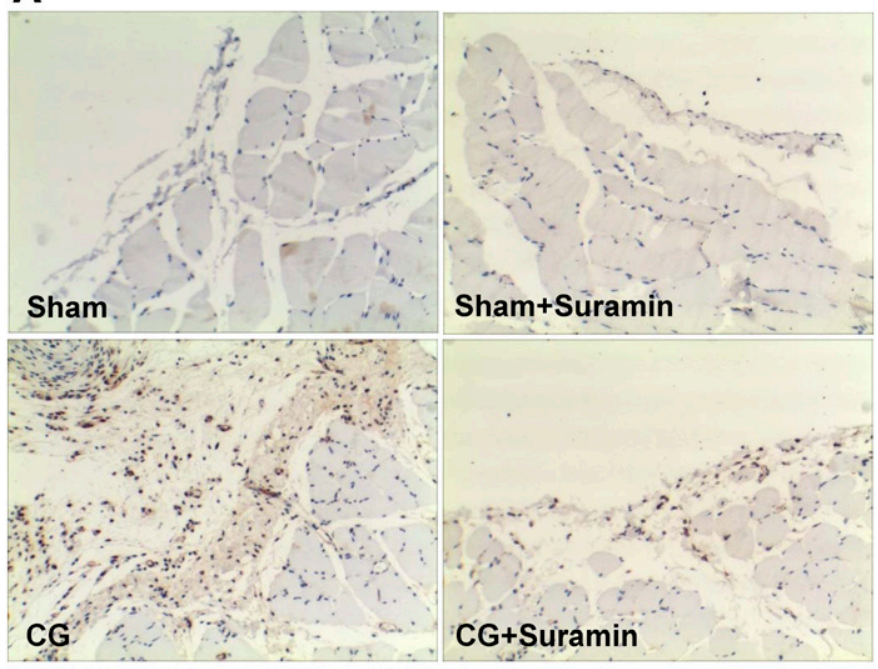

B

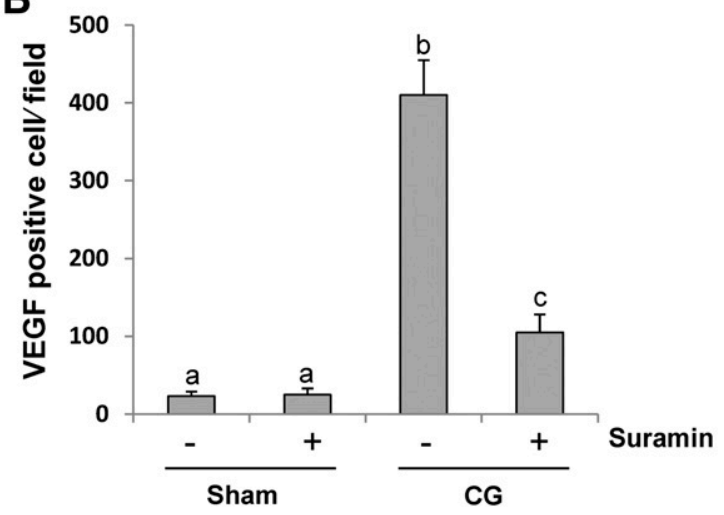

Fig. 10. Effect of suramin on the peritoneal expression of VEGF in the rat model of CG-induced peritoneal fibrosis. The protein expression of VEGF was detected by immunohistochemistry staining (A). Quantitative analysis of peritoneal VEGF expression in three random fields of each sample, and 10 fields $(1 \times 100)$ were analyzed for each condition (B). Data are represented as the mean \pm S.E.M. $(n=6)$. Means with different lowercase letters are significantly different from one another $(P<0.05)$

suramin inhibits phosphorylation of epidermal growth factor receptor and platelet-derived growth factor receptors, two growth factor receptors associated with tissue fibrosis in various organs, including peritoneum. Further studies are needed to investigate the effect of suramin on the activation of these two receptors in peritoneal fibrosis.

It is well documented that inflammation contributes to peritoneal fibrosis. Inflammatory response is characterized by expression of multiple cytokines/ chemokines and infiltration of proinflammatory cells, in particular macrophages. In this study, we demonstrated that suramin treatment inhibits expression of proinflammatory cytokines/chemokines, such as IL-1, IL-6, TNF- $\alpha$, and MCP-1, and infiltration of macrophages. As such, it is likely that another mechanism of suramin inhibition of peritoneal fibrosis is through suppression of inflammation in the injured peritoneum. In support of this speculation, we found that suramin inhibits phosphorylation of NF- $\kappa \mathrm{Bp} 65$, a key transcriptional factor for the expression of multiple proinflammatory chemokines and cytokines and phosphorylation of 
$\mathrm{p}-\mathrm{I} \kappa \mathrm{B} \alpha$. Because $\mathrm{I} \kappa \mathrm{B} \alpha$ is an inhibitor of NF- $\kappa \mathrm{B}$ signaling and its phosphorylation is required for activation of NF- $\kappa \mathrm{B}$ activation, inhibition of $\mathrm{I} \kappa \mathrm{B} \alpha$ phosphorylation would contribute to inactivation of NF- $\kappa \mathrm{B}$ signaling and subsequent inhibition of transcription of inflammatory genes.

Angiogenesis and vasculopathy in the peritoneum play an important role in the regulation of water and solute transport in the peritoneum (Williams et al., 2002). However, increased vascularity and/or peritoneal blood flow resulted in progressive peritoneal fibrosis (Davies et al., 2011). This manifests itself as submesothelial thickening and is associated with increased vascularization that leads to ultrafiltration dysfunction (Sekiguchi et al., 2012). Abundance of in vitro and in vivo studies (Margetts et al., 2001; Ghosh and Karin, 2002) have shown that vascular endothelial growth factor induced by TGF- $\beta 1$ can upregulate the expression of VEGF receptor 1 and interaction of VEGF with VEGF receptor 1 leads to angiogenesis and ultimately ultrafiltration failure. On this basis, we speculated that reduction of VEGF expression would alleviate angiogenesis and improve ultrafiltration of peritoneal membrane. In the present study, we examined the effect of suramin on the expression of VEGF and found that suramin was effective in suppressing its expression in rat peritoneum after chlorhexidine gluconate injection. In addition, we also observed that suramin treatment inhibited expression of CD31, a marker of vascular endothelial cells (Fig. 11). Therefore, suramin may inhibit neo-angiogenesis and protect the peritoneum from fibrosis by suppressing production of angiogenic growth factors.

PD has been widely accepted as a treatment of end-stage renal disease patients. Success of this modality is largely dependent on the structural and functional integrity of the peritoneal membrane. However, the duration of PD can be limited by peritoneal alteration in both structure and function, including peritoneal fibrosis and ultrafiltration failure (Saxena, 2008; Davies et al., 2011). In vitro and in vivo studies (Liu et al., 2014) have demonstrated that peritoneal fibrosis is characterized by detachment of the mesothelial layer, dilatation of intercellular spaces, and deposition of extracellular matrix (Devuyst et al., 2010). Our current studies have also shown that suramin can inhibit submesothelial thickening, deposition of fibrils, and expression of multiple ECM proteins, suggesting its usefulness in attenuating peritoneal fibrosis and reducing ultrafiltration failure. Given the fact that there is no available therapy for halting or preventing this complication thus far, it would be interesting to conduct clinical trials in the future to investigate whether suramin can be used as an additive component in the $\mathrm{PD}$ dialysate for preventing development of peritoneal fibrosis during PD.

However, there are two limitations for this study. First, suramin administration only started immediately after injection of chlorhexidine gluconate, the inducer of peritoneal fibrosis. As such, the current results only suggest that suramin is an effective agent that prevents development of peritoneal fibrosis, but they do not show whether it also has a therapeutic effect in established peritoneal fibrosis. To gain more information about its therapeutic effect, it is necessary to administer suramin at different time points after injection of chlorhexidine gluconate to study its effects on peritoneal fibrosis. Second, we only examined the antifibrotic effect of suramin in one animal model of peritoneal fibrosis. Because peritoneal fibrosis is a complex process that is caused by
A

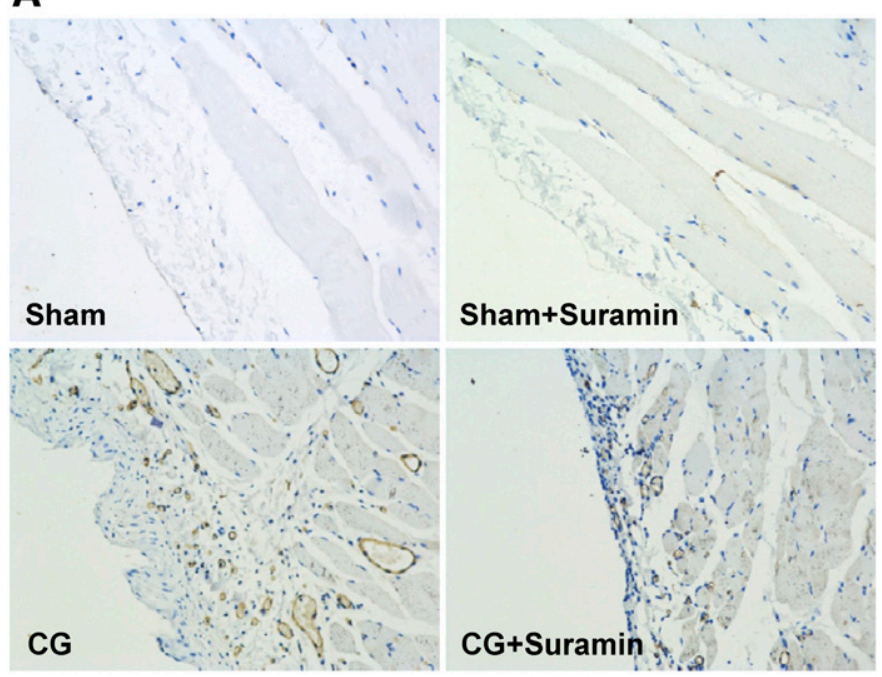

B CD31

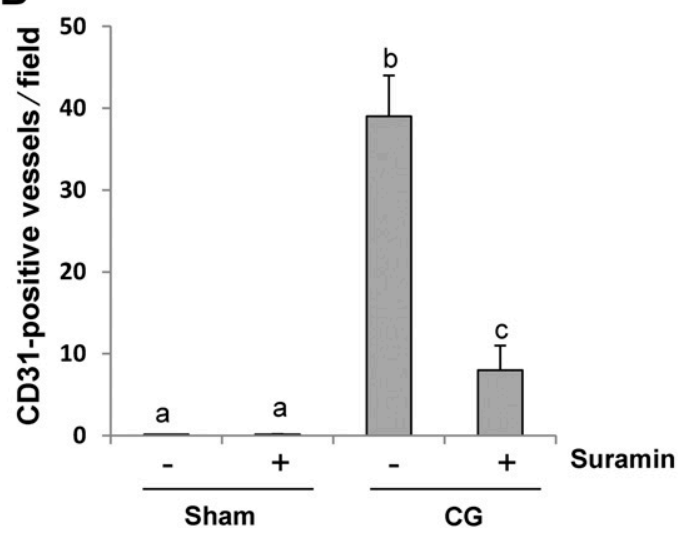

Fig. 11. Effect of suramin on peritoneal angiogenesis in the rat model of CG-induced peritoneal fibrosis. The protein expression of CD31 was detected by immunohistochemistry staining (A). Quantitative analysis of peritoneal CD31 expression in three random fields of each sample, and 10 fields $(1 \times 100)$ were analyzed for each condition $(B)$. Data are represented as the mean \pm S.E.M. $(n=6)$. Means with different lowercase letters are significantly different from one another $(P<0.05)$.

multiple pathogenic factors and is involved in different mechanisms, it is possible that the antifibrotic effect of suramin may vary with animal models of this disease. Therefore, additional animal studies with delayed administration of suramin in different models of peritoneal fibrosis are required prior to initiation of a clinical trial to test its effectiveness in human peritoneal fibrosis.

In summary, we demonstrate that suramin is able to prevent the development of peritoneal fibrosis. The antifibrotic effect of suramin is associated with inactivation of TGF- $\beta 1$ and NF- $\kappa$ B signaling, inhibition of macrophage infiltration and cytokine/chemokine expression, as well as suppression of angiogenesis. As peritoneal fibrosis is involved in the increased production of numerous cytokines/growth factors and subsequent activation of their receptors and signaling pathways, suramin, with its ability to inhibit the interaction of multiple cytokines/growth factors with their receptors, may have therapeutic potential in the treatment of peritoneal fibrosis. Thus, suramin may become a novel therapeutic approach to prevent the development of peritoneal fibrosis in PD patients. 


\section{Authorship Contributions}

Participated in research design: Yan, Liu, Zhuang.

Conducted experiments: Xiong, Liu.

Contributed new reagents or analytic tools: Xiong, Yan, Liu,

Zhuang.

Performed data analysis: Xiong, Fang, Liu.

Wrote or contributed to the writing of the manuscript: Xiong, Fang,

Yan, Liu, Zhuang.

\section{References}

Aroeira LS, Aguilera A, Selgas R, Ramírez-Huesca M, Pérez-Lozano ML, Cirugeda A Bajo MA, del Peso G, Sánchez-Tomero JA, and Jiménez-Heffernan JA, et al. (2005) Mesenchymal conversion of mesothelial cells as a mechanism responsible for high solute transport rate in peritoneal dialysis: role of vascular endothelial growth factor. Am J Kidney Dis 46:938-948.

Bhogal RK, Stoica CM, McGaha TL, and Bona CA (2005) Molecular aspects of regulation of collagen gene expression in fibrosis. J Clin Immunol 25:592-603.

Blake PG, Jain AK, and Yohanna S (2013) Biocompatible peritoneal dialysis solutions: many questions but few answers. Kidney Int 84:864-866.

Bozkurt D, Taskin H, Sezak M, Biçak S, Sen S, Ok E, and Duman S (2008) Rosiglitazone, a peroxisome proliferator-activated receptor agonist, improves peritoneal alterations resulting from an encapsulated peritoneal sclerosis model. $A d v$ Perit Dial 24:32-38.

Chan YS, Li Y, Foster W, Horaguchi T, Somogyi G, Fu FH, and Huard J (2003) Antifibrotic effects of suramin in injured skeletal muscle after laceration. $J$ Appl Physiol (1985) 95:771-780

Chaudhary K, Sangha H, and Khanna R (2011) Peritoneal dialysis first: rationale. Clin J Am Soc Nephrol 6:447-456.

Chegini N (2008) TGF-beta system: the principal profibrotic mediator of peritoneal adhesion formation. Semin Reprod Med 26:298-312.

Davies SJ, Mushahar L, Yu Z, and Lambie M (2011) Determinants of peritoneal membrane function over time. Semin Nephrol 31:172-182.

de Lima SM, Otoni A, Sabino AdeP, Dusse LM, Gomes KB, Pinto SW, Marinho MA and Rios DR (2013) Inflammation, neoangiogenesis and fibrosis in peritoneal dialysis. Clin Chim Acta 421:46-50.

De Vriese AS, Tilton RG, Stephan CC, and Lameire NH (2001) Vascular endothelial growth factor is essential for hyperglycemia-induced structural and functional alterations of the peritoneal membrane. J Am Soc Nephrol 12:1734-1741.

Devuyst O, Margetts PJ, and Topley N (2010) The pathophysiology of the peritoneal membrane. J Am Soc Nephrol 21:1077-1085.

Fusshoeller A (2008) Histomorphological and functional changes of the peritoneal membrane during long-term peritoneal dialysis. Pediatr Nephrol 23:19-25.

Ghosh S and Karin M (2002) Missing pieces in the NF-kappaB puzzle. Cell 109 (Suppl):S81-S96.

Herrmann J, Ciechanover A, Lerman LO, and Lerman A (2004) The ubiquitinproteasome system in cardiovascular diseases-a hypothesis extended. Cardiovasc Res 61:11-21.

Hoesel B and Schmid JA (2013) The complexity of NF- $\mathrm{kB}$ signaling in inflammation and cancer. Mol Cancer 12:86.

Huang H, Liu Y, Daniluk J, Gaiser S, Chu J, Wang H, Li ZS, Logsdon CD, and Ji B (2013) Activation of nuclear factor-кB in acinar cells increases the severity of pancreatitis in mice. Gastroenterology 144:202-210.

Kaminska B, Wesolowska A, and Danilkiewicz M (2005) TGF beta signalling and its role in tumour pathogenesis. Acta Biochim Pol 52:329-337.

Kaneko K, Hamada C, and Tomino Y (2007) Peritoneal fibrosis intervention. Perit Dial Int 27 (Suppl 2):S82-S86.

Karin M (2009) NF-kappaB as a critical link between inflammation and cancer. Cold Spring Harb Perspect Biol 1:a000141.

Kitamura M, Nishino T, Obata Y, Furusu A, Hishikawa Y, Koji T, and Kohno S (2012) Epigallocatechin gallate suppresses peritoneal fibrosis in mice. Chem Biol Interact 195:95-104.

Lai KN and Leung JC (2010) Inflammation in peritoneal dialysis. Nephron Clin Pract 116:c11-c18.

Lee CJ, Subeq YM, Lee RP, Liou HH, and Hsu BG (2014) Calcitriol decreases TGF- $\beta 1$ and angiotensin II production and protects against chlorhexide digluconateinduced liver peritoneal fibrosis in rats. Cytokine 65:105-118.
Li C, Jiang X, Yang L, Liu X, Yue S, and Li L (2009) Involvement of sphingosine 1-phosphate (SIP)/S1P3 signaling in cholestasis-induced liver fibrosis. Am J Pathol 175:1464-1472.

Liu N and Zhuang S (2011) Tissue protective and anti-fibrotic actions of suramin: new uses of an old drug. Curr Clin Pharmacol 6:137-142.

Liu N, Tolbert E, Pang M, Ponnusamy M, Yan H, and Zhuang S (2011) Suramin inhibits renal fibrosis in chronic kidney disease. J Am Soc Nephrol 22:1064-1075.

Liu Q, Zhang Y, Mao H, Chen W, Luo N, Zhou Q, Chen W, and Yu X (2012) A crosstalk between the Smad and JNK signaling in the TGF- $\beta$-induced epithelialmesenchymal transition in rat peritoneal mesothelial cells. PLoS ONE 7:e32009.

Liu Y (2006) Renal fibrosis: new insights into the pathogenesis and therapeutics. Kidney Int 69:213-217.

Liu Y, Li JF, Liu H, Liu FY, Peng YM, Liu YH, Cheng MC, Chen GC, and Zhou X (2014) Functional and structural alterations of peritoneum and secretion of fibrotic cytokines in rats caused by high glucose peritoneal dialysis solutions. Ren Fail 36: $292-299$.

Man J and Zhang X (2011) CUEDC2: an emerging key player in inflammation and tumorigenesis. Protein Cell 2:699-703.

Margetts PJ, Kolb M, Galt T, Hoff CM, Shockley TR, and Gauldie J (2001) Gene transfer of transforming growth factor-beta1 to the rat peritoneum: effects on membrane function. J Am Soc Nephrol 12:2029-2039.

Muriel P (2009) NF-kappaB in liver diseases: a target for drug therapy. $J$ Appl Toxicol 29:91-100.

Nakamura S and Niwa T (2004) Advanced glycation end-products and peritoneal sclerosis. Semin Nephrol 24:502-505.

Nishino T, Ashida R, Obata Y, Furusu A, Abe K, Miyazaki M, Koji T, and Kohno S (2012) Involvement of lymphocyte infiltration in the progression of mouse peritoneal fibrosis model. Ren Fail 34:760-766.

Pang M, Kothapally J, Mao H, Tolbert E, Ponnusamy M, Chin YE, and Zhuang S (2009) Inhibition of histone deacetylase activity attenuates renal fibroblast activation and interstitial fibrosis in obstructive nephropathy. Am J Physiol Renal Physiol 297:F996-F1005.

Patel P, Sekiguchi Y, Oh KH, Patterson SE, Kolb MR, and Margetts PJ (2010) Smad3-dependent and -independent pathways are involved in peritoneal membrane injury. Kidney Int 77:319-328.

Ro Y, Hamada C, Inaba M, Io H, Kaneko K, and Tomino Y (2007) Inhibitory effects of matrix metalloproteinase inhibitor ONO-4817 on morphological alterations in chlorhexidine gluconate-induced peritoneal sclerosis rats. Nephrol Dial Transplant 22:2838-2848.

Saxena R (2008) Pathogenesis and treatment of peritoneal membrane failure. Pediatr Nephrol 23:695-703.

Sekiguchi Y, Zhang J, Patterson S, Liu L, Hamada C, Tomino Y, and Margetts PJ (2012) Rapamycin inhibits transforming growth factor $\beta$-induced peritoneal angiogenesis by blocking the secondary hypoxic response. J Cell Mol Med 16: 1934-1945.

Stavenuiter AW, Schilte MN, Ter Wee PM, and Beelen RH (2011) Angiogenesis in peritoneal dialysis. Kidney Blood Press Res 34:245-252.

Subeq YM, Ke CY, Lin NT, Lee CJ, Chiu YH, and Hsu BG (2011) Valsartan decreases TGF- $\beta 1$ production and protects against chlorhexidine digluconate-induced liver peritoneal fibrosis in rats. Cytokine 53:223-230.

Sun M, Dawood F, Wen WH, Chen M, Dixon I, Kirshenbaum LA, and Liu PP (2004) Excessive tumor necrosis factor activation after infarction contributes to susceptibility of myocardial rupture and left ventricular dysfunction. Circulation 110:3221-3228.

Tomino Y (2012) Mechanisms and interventions in peritoneal fibrosis. Clin Exp Nephrol 16:109-114.

Yoshio Y, Miyazaki M, Abe K, Nishino T, Furusu A, Mizuta Y, Harada T, Ozono Y, Koji $\mathrm{T}$, and Kohno S (2004) TNP-470, an angiogenesis inhibitor, suppresses the progression of peritoneal fibrosis in mouse experimental model. Kidney Int 66:1677-1685. Williams JD, Craig KJ, Topley N, Von Ruhland C, Fallon M, Newman GR, Mackenzie RK, and Williams GT; Peritoneal Biopsy Study Group (2002) Morphologic changes in the peritoneal membrane of patients with renal disease. J Am Soc Nephrol 13:470-479. Wynn TA (2008) Cellular and molecular mechanisms of fibrosis. J Pathol 214:199-210.

Address correspondence to: Dr. Haidong Yan, Department of Nephrology, Tongii University School of Medicine, 150 Ji Mo Road, Pudong New District, Shanghai 200120, China. E-mail: yhdcmu@sina.com; or Dr. Shougang Zhuang, Brown University School of Medicine, Rhode Island Hospital, Middle House 301, 593 Eddy Street, Providence, RI 02903. Email: szhuang@lifespan.org 